\title{
Field level study to understand dimensions of antimicrobial use in dairy farms of Punjab
}

\author{
Neela Madhav Patnaik, Jancy Gupta and BS Meena
}

Received: 30 January 2020 / Accepted: 09 June 2020 / Published online: 27 October 2020

(c) Indian Dairy Association (India) 2020

\begin{abstract}
As a disease control measure on dairy farms for diseased animals, ensuring prudent antimicrobial use is a necessary step. In this perspective, Antimicrobial Resistance (AMR) has been an emerging global threat cutting across boundaries which is a serious threat to humans and dairy animals. Thus research on antimicrobial use practices and veterinarian prescribing behaviour is critical in achieving national efforts of managing AMR as far as dairy sector is concerned. In this context of antimicrobial use in dairy farming, the study was conducted in Punjab state of Northern India. Multistage stratified random sampling technique was applied to get the first hand information from 180 farmers and 60 veterinary doctors. None of the dairy farmers in the study area were following withdrawal period after their sick animal was administered with antimicrobial. Garett method of ranking on importance of treatment sources by respondents ranked the veterinary doctor first position with paravet ranked second. Prescribing behaviour of the veterinary doctors revealed prior experience of treatment and prompt results i.e. quick relief to animals as decisive factor in antimicrobial administration. Empirical research findings on dimensions of antimicrobial use in dairy farms can be vital in designing future strategies for tacking AMR in dairy sector.
\end{abstract}

Dairy Extension Division, ICAR-National Dairy Research Institute, Karnal-132 001, India

BS Meena $(\bowtie)$

Dairy Extension Division, ICAR-National Dairy Research Institute, Karnal-132 001, India

Email: bmeena65@yahoo.co.in
Keywords: Antimicrobials, Consultation, Dairy Animal, Farmer, Treatment

\section{Introduction}

A developing country like India has the highest bacterial infections as a result of which antibiotic use is widespread and indiscriminate; acting as a driving force towards resistance issue among dairy animals (Kuralayanapalya et al. 2019). Food producing animals which include dairy animals act as prime reservoir of zoonotic pathogens which pose severe health risk to humans. Resistant pathogenic strains in dairy animals are Staphylococcus aureus, Salmonella spp., Escherichia coli, Listeria monocytogenes etc. often contaminating milk and milk products. S. aureus is frequently associated in case of mastitis which leads to its entry in the milk food chain (Sharma et al. 2018). In dairy sector, antibiotics are excessively used in treating intramammary infections which affects the quality of milk produced due to presence of antibiotic residues. This residue contaminated milk may induce intestinal alterations and allergies subsequently resulting in emergence of multidrug resistant bacteria among milk consumers (Sandholm et al. 2009). Several studies conducted from different areas of India have reported the presence of antimicrobial residues in milk which indicates the widespread antimicrobial use in dairy production systems (Moudgil et al. 2019; Lindahl, et al. 2018; Kurjogi et al. 2019). The adverse effect of residues present in milk is seen in manufacturing of dairy products like cheese and yogurt as the residues inhibit microflora of milk (Aalipour et al. 2013). National Health Policy (2017) has reiterated the lack of stringent regulations against non-therapeutic use of antibiotics. The overuse of antibiotics in animal sector has led to AMR emergence which is an unmeasured burden on India. The Policy document has identified AMR as a severe problem which requires effective actions in addressing it. By 2050, estimated economic burden due to AMR could be around $\$ 100$ trillion and mortality numbers globally would be 10 million, with Asia alone contributing almost half of them at 4.7 million (O’Neill, 2018).

AMR as a global health issue can be gauged from the fact that UN high level meeting in the year 2016 focussed exclusive discussion on AMR. It was a rare instance as it was only the 
fourth time a health issue was discussed after HIV, Noncommunicable disease and EBOLA. In this perspective of tackling AMR, India has acted proactively by devising National Action Plan (NAP) for AMR.

NAP-AMR has outlined 5 strategic priorities to be implemented over 2017-2021 in tackling health challenge by AMR in India.

1. Improve awareness and understanding of AMR through effective communication, education and training

2. Strengthen knowledge and evidence through surveillance

3. Reduce the incidence of infection through effective infection prevention and control

4. Optimize the use of antimicrobial agents in health, animals and food

5. Promote investments for AMR activities, research and innovations

A closer look to the above five strategies from the social science lens highlights the importance of survey research in extracting the field level information from different stakeholders (farmers being primary stakeholder) with much emphasis towards dairy health care management practices. This baseline data generated can prove to be a cornerstone in plugging the gaps in tackling the AMR issue strategically in achieving the five priorities.

Kakkar et al. (2017) had opined the importance of smaller studies at regional levels in understanding the antibiotic consumption and resistant pattern due to lack of awareness on AMR and surveillance programs at national level. Though the smaller studies may not provide full understanding of the problem, but with combined efforts can act as systematic surveillance among farmers and dairy professionals in reducing antibiotic misuse. Overall research in the area of antimicrobial resistance has been limited in social science field and recently research from the farmer's perspective has gained momentum to understand the critical factors underlying AMR. Veterinary doctors inappropriate antimicrobial prescription can trigger selective pressure on bacterial infections thus increasing AMR of microorganisms in animals transferring to humans through the food chain. A number of extrinsic and intrinsic factor influence veterinarian prescribing behaviour. Norris et al. (2019) stated drug preference, professional experience, ease of administration, animal characteristics, perceived antimicrobial efficacy and cost of diagnostic tests.

Patnaik et al.(2019) on their study on veterinary doctors of Punjab reported the factors limiting them from undergoing sensitivity testing were sampling difficulties of the infections, the urgency of the situation for prescribing antimicrobial and concerns regarding the clinical relevance of in vitro tests.
The prescribing behaviour of vets engaged in treating dairy animals assumes much importance in the current antimicrobial use and resistance scenario.

To raise awareness regarding AMR concerns among dairy stakeholders, it is imperative to collect data across different regions of the country regarding antimicrobial usage and treatment patterns. The present research can help in developing evidence based policy towards regulating antimicrobial use at farmer's level. Exploring the dimensions of antimicrobial use in dairy farms through surveying farmers and veterinary doctor perspective; the two major stakeholders of the study forms the crux of this study.

\section{Materials and Methods}

The present study conducted towards understanding the field level practices that are facilitating towards AMR has followed a reductionist tradition of science. The survey research was conducted in purposively selected state of Punjab. Research design utilized for the study was both descriptive and exploratory. Multistage random sampling was followed for the study in selection of district, block, village and final respondents of the study. Three randomly districts selected for the study were Amritsar, Ludhiana and Pathankot and from each district two blocks were selected and from each block two villages. 15 farmers from each village were selected by stratified random sampling, thus totalling to 180 farmers for the study. The inclusion criterion for the farmer to be part of the study was that they should rear at least one milch animal for the last five years. The first-hand information on consultation and follow-up treatment practices on antimicrobial resistance in milk was gleaned from 180 respondents with the help of semi-structured interview schedule. Focus Group Discussions were carried out and oral histories were recorded in field diary to explore the various dimensions facilitating antimicrobial use in dairy farms at file level. Veterinary doctors working at the block level under the Govt. of Punjab providing service to dairy farmers in the selected villages were chosen for the study. A total of 60 veterinary doctors constituted as respondents of the study with 20 block level veterinarians from each district selected purposively. The data was analysed using statistical tools such as Frequency, Percentage and Garett ranking method to draw meaningful conclusions represented by stacked bar graphs, pie charts and tables.

\section{Garett ranking method}

In social science studies, Garett ranking method is widely used to rank the response data based on importance generated by survey research.

For converting ranks into percent position, the garett formula is Per cent position $=100 *\left(\mathrm{R}_{\mathrm{ij}}-0.5\right) / \mathrm{N}_{\mathrm{j}}$ 
Where,

$\mathrm{R}_{\mathrm{ij}}$ is the rank given for $\mathrm{i}^{\text {th }}$ factor by $\mathrm{j}^{\text {th }}$ individual

$\mathrm{N}_{\mathrm{j}}$ is the number of factors ranked by the $\mathrm{j}^{\text {th }}$ individual

With the help of conversion table given by Garrett and Woodworth (1969), the percent position scores of each rank are converted into garett values. The next step is adding together the individual respondent's score for each factor and then dividing it by total number of respondents. The obtained mean scores for all the factors are arranged and ranked in descending order to identify the important ones.

\section{Results and Discussion}

\section{Management practices for diseased animals by dairy farmers}

Effective recovery of diseased animals requires certain precautions and practices to be undertaken so that the disease does not transmit to healthy animals in the herd. Isolating the sick animals is usually recommended along with disinfection of shed at regular intervals to curb disease incidence and transmission. A multiple choice question was framed to investigate the precaution taken by dairy farmers for diseased animals. The four options were isolating sick animal after being treated; disinfecting the animal shed; both isolation the animal and disinfecting the shed; none of the above practices were followed.

A perusal of Fig.1 revealed that 17 percent farmers were isolating their treated diseased animal whereas disinfecting the shed was carried out by 22 percent of the respondents in the study area. In India most of the dairy farmers are usually small and marginal in nature and dairy animals are reared with great affection and emotional bond exists between the animal and members of the farmer family. Those dairy farmers who were not isolating their treated sick animals put forward a number a reasons. They were lack of space, difficulty in providing feed and water separately to healthy and sick animals and the animals were reared within the compound of farmer house. The researchers observed that farmers possessing small herd size were also acting as a hindrance for isolating the animal. But the main issue was the farmers were unaware of the benefits of isolating the animal to protect the healthy animals getting infected. The respondents opined that isolating the animals separately would further worsen their health indicating the compassion showed by farmers towards their herd. About 41 percent reported of following both isolating the sick animals and disinfecting the shed regularly. These respondents were possessing medium-large herds of 10-20 animals having separate animal shed. Education and extension contact played a crucial role in farmers awareness and know how following the practices of isolation and disinfection of shed for better recovery of diseased animal and preventing the spread of disease to the healthy animals of the herd.

\section{Methods of treatment carried for dairy animals ailments}

In case of diseased animals, dairy farmers have a number of choices of various treatment methods available depending on according to the financial capacity, severity of the disease and promptness of the curing methods. The different curing methods have certain bearing as far as AMR issues are concerned though homeopathy and allopathic treatment are considered novel methods. Dairy farmers prefer allopathic treatment for their animals in most cases as they are prompt in curing diseases and provide quick relief though the overuse/sub optimal use of antimicrobials can result in resistant to infections. Majority $(90 \%)$ of dairy farmers frequently preferred allopathic treatment for treatment of their animals as shown in Fig.2. As reported by dairy farmers, ayurveda and homeopathy were never a preferred choice for treatment of dairy animals. Focus group discussion conducted with dairy farmers revealed that those possessing indigenous buffaloes and cattle were more resistant to disease infections. During the interaction with farmers, few innovative and progressive farmers stated that they had tried ayurveda and homeopathic treatment for their animals but they were largely unsuccessful and their efficacy was quite low compared to allopathic treatment. Nair et al. (2015) reported the utility of ayurveda treatment practices as it reduced the number of antibiotic positive milk samples by 18 to $49 \%$ in India. Chand et al. (2016) in their study to compare the efficacy of homeopathic and allopathic treatments against Foot and Mouth disease in cattle reported that treatment of FMD affected animals with homeopathic drugs (Kalium iodatum, Calendula, and Sulphur) led to better recovery of animals in terms of reducing temperature, increasing appetite and ruminal motility, and rapid healing of the oral mucosal and foot lesions. Hence it is suggested that similar comparative research studies are needed in cases of other diseases of milch animals so that ayurveda and homeopathy can act as potential alternative treatment for animals against prevailing allopathic treatment practices. The visibility and scope of alternate treatment methods are highly necessary to reduce to reliance on antibiotic use thus minimizing the residues in milk making it safe for human consumption.

\section{Treatment guidelines follow-up by dairy farmers}

To understand the aspects farmers were following after their dairy animal is treated with antimicrobials helps to know the gaps between the recommended practices and actual practice taking place at field level. The farmers were asked on a 3 point continuum regarding three crucial after treatment recommended practices of discarding the milk of treated animal(withdrawal period), purchasing the full recommended dose and completing the course of antimicrobial. Surprisingly, in the study area none of the farmers were following withdrawal period i.e. discarding the milk of treated animal (Fig. 3). Withdrawal period is the time between the last dose of antimicrobial treated and the time when the milk can be safely consumed. Following the withdrawal period reduces the 
Fig.1 Management practices for diseased animals by dairy farmers

Precaution for sick animals

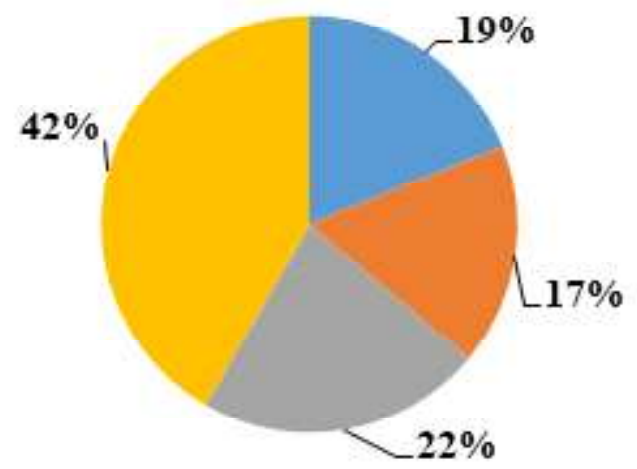

None

Isolate sick animal(s)

Disinfect animal shed

Both isolation \& disinfection

Fig.2 Methods of treatment carried for dairy animals ailments

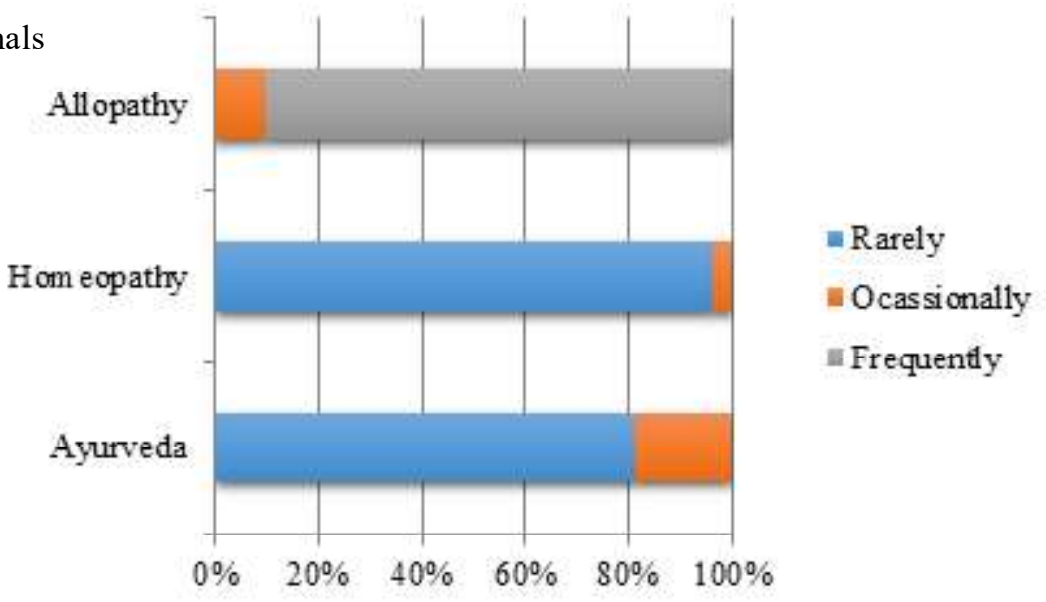

Fig.3 Treatment guidelines follow-up by dairy farmers

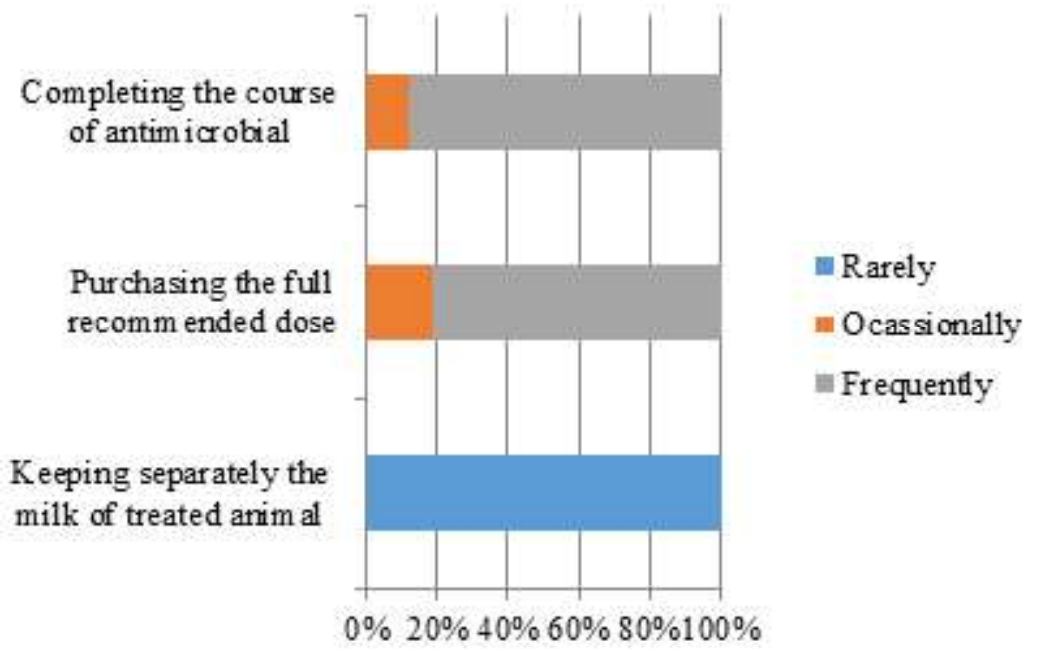

drug to safe tolerance levels so that residues in milk in not consumed by humans. The researchers observed that the farmers were unaware regarding withdrawal period. The researchers informed the farmers regarding the ill effects of residues in milk and benefit of following withdrawal period. The farmers reiterated that following withdrawal period was not feasible as they would incur heavy financial loss by discarding the milk. An open ended question was asked to farmers whether they would follow the withdrawal period if premium price would be provided for residue free milk by milk co-operatives. The question provided mixed 
Fig.4 Prescribing behaviour of antimicrobials by veterinarians

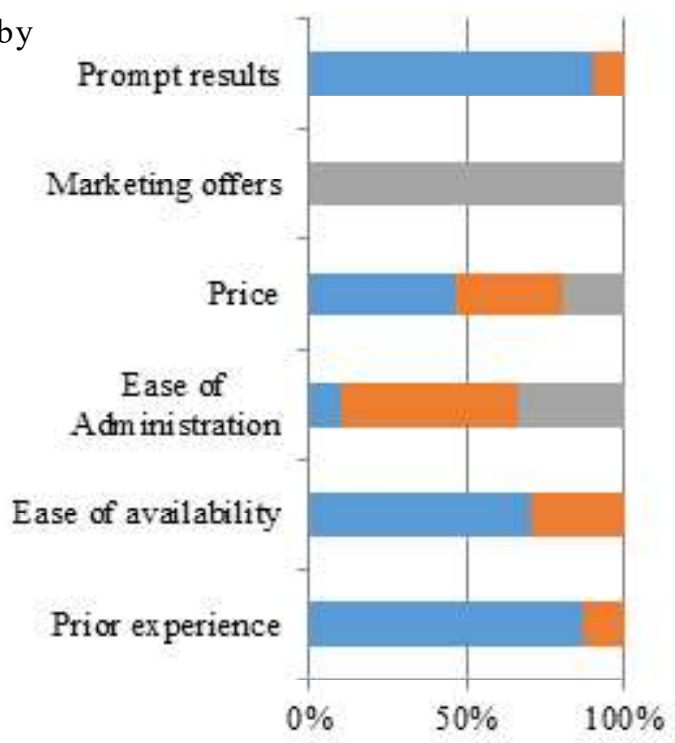

- Most Im portant

Esomewhat Im portant
Table 1 Ranking of treatment sources based on their credibility

\begin{tabular}{llc}
\hline Sources of Treatment & Mean Garett Score & Rank \\
\hline Veterinary Doctor & 72.13 & 1 \\
Paravet & 61.40 & 2 \\
Over - the- Counter(OTC) & 46.72 & 3 \\
Private Milk vendor & 41.63 & 4 \\
Other Dairy farmer & 39.08 & 5 \\
Milk Co-Operative official & 37.01 & 6 \\
\hline
\end{tabular}

reaction from the farmers. The farmers with large herds agreed to the proposition of following the withdrawal period if such a policy would be formulated. Small farmers were not interested in proposition of receiving premium price for residue free milk as most of them sold milk in the unorganized sector. Eltayb et al. (2012) reported a technique that by following pasteurization and electrochemical oxidation of raw milk with oxytetracycline at 100 $\mathrm{mg} / \mathrm{ml}$, milk of antibiotic treated animal can be made suitable for feeding calves. Practically this procedure is difficult at farmer level due to lack of awareness and know-how and further research is needed to make the above technology accessible to the farmers and understanding the efficacy of the technique.

A perusal of Fig. 3 reveals that 93.70 percent of the respondents reported of purchasing the full recommended of medicines prescribed. The farmers consulting the veterinarians for treating animal diseases charged fees which include the antibiotics provided to them during and after the treatment. It was observed that 90.74 percent of farmers reported of completing the antimicrobial course prescribed for their dairy animals. A critical observation of the results shows 93.70 percent purchasing the full recommended dose and 90.74 percent completing the prescribed antimicrobial course. This difference can be attributed to the cases of farmers using left over medicines in subsequent cases of similar diseases or ailments. In depth discussion with the farmers, it was observed that farmers were saving medicines so that they could be utilized later. A study by Sawant et al. (2005) found only 24 percent of respondents completing the antimicrobial prescribed course; thus failure in completion the course could lead to sub-optimal use of antimicrobials which is a major factor of AMR

\section{Ranking of treatment sources based on their credibility}

Treatment to animals of dairy farmer is provided by number of persons or sources. Understanding the credibility associated with each treatment source helps us to know whom the farmer trust regarding the health care management practices of diseased animal. Six treatment sources were identified after consulting dairy experts and pilot study with farmers of non-sampling area. The respondents were asked to rank the treatment sources in ascending order from 1 to 6 based on the credibility of treatment sources in health care management aspect. Garett ranking method was employed to analyse the ranking data generated from the respondents. Veterinary doctor was ranked first position by the dairy farmers as the most credible source of providing treatment (Table. 1). The farmers reported of consulting the veterinarian when their animal gets sick. Bergevoet (2019) studied the antibiotic use in Dutch farms; found the sow farmers considered veterinarian followed by feed supplier as the most important source of information on antibiotic use and treatment. Similar studies conducted on antibiotic use in farms from different regions have invariably reported the veterinarian to be the most crucial treatment source for diseased animals among various treatment sources (Ison and Rutherford, 2014; Jones et al. 2015). Paravets were ranked the second position which can be attributed due to their personal cosmopoliteness. The researchers found that farmers of Pathankot district were possessing small herd of dairy animals and were more dependent on paravets for treating their animals. The paravets were easily assesible to farmers and 
charged less compared to veterinary doctors. Due to lack of strict guidelines, availability of antibiotics over the counter (OTC) was widely prevalent in the study area. Hence the OTC persons were ranked third by the farmers. Self-prescription was also seen in case of progressive farmers, who have gained skills in treating the animals with years of experience in dairy farming. The farmers selling milk in the unorganized sector to milk vendors (dudias) reported they consulted sometimes vendors in case of minor ailments. A good observation by the researchers which reflects in the rankings was the low credibility of vendors and milk cooperative persons in providing treatment advice to farmers. These sources of treatment are unreliable and consulting them would result in misuse of antibiotics. The high credibility of veterinary doctor can act as the most important stakeholder in changing the behaviour of farmers towards antibiotic use. It would be highly useful to include the veterinarian and possibly the paravet in creating self-awareness regarding AMR issue and disseminating information to farmers by undertaking improved and easy practices of maintain hygiene and cleaning the shed regularly and vaccination the animals regularly. These small steps can have a large effect in controlling the disease in animals ultimately reducing the overall antibiotic use.

\section{Prescribing behaviour of antimicrobials by veterinarians}

Veterinary doctors are an indispensable part of the research on antimicrobial use because of the treatment they provide to the sick dairy animals. The veterinarians were asked to respond to the statements on factors of prescribing behaviour on a 3 point continuum of importance they felt towards each statement. The two prominent factors were prompt result i.e. quick relief to sick animals and prior experience of drugs and treating similar diseases. A perusal of Fig. 4 indicates that 90 percent of respondents stated prompt result is a decisive factor for prescribing antimicrobials whereas prior experience was stated by 86 percent respondents. The importance given to prior experience in choosing and administering the antimicrobial by vets can be attributed to their knowledge on the aetiological agent and its susceptibility profile. Gibbons et al. (2013) in their study on veterinary practioners of Ireland found that 95.7 percent of the respondents considered prior experience of treatment 'often' or 'always' in making decisions on prescribing drugs. Ease of availability of antimicrobial was also kept in mind while administering or prescribing antimicrobial by 70 percent of the respondents. Providing quick relief to the diseased animals was the prime motto perceived by the veterianians, hence price of the antimicrobial was not that a decisive factor. Still 46 percent of the respondents had considered the price of antimicrobial while prescribing them. The veterinarians stated marketing offers by various marketing representatives have never influenced their decision of prescribing antimicrobial. Veteriarians in the study area reported the antimicrobials they frequently prescribed for treating various diseases of dairy animals were Ceftiofur, Oxytetracycline, Ceftriazone, Gentamycin, Enrofloxacin,
Sulphadimiene, Marbofloxacin, Ceftizozim, Streptopenicilin, Methoxazole etc. Briyne et al. (2013) reported the factors strongly influencing prescribing behaviour of veterinarians in Europe were ease of administration, risk of developing antibiotic resistance, sensitivity tests and experience of the respondents.

\section{Conclusions}

Field study was undertaken to understand dimensions of antimicrobial use in dairy farms which is very crucial to formulate policies if at all the risk due to antimicrobial resistance is to be tackled. Allopathic treatment was mostly preferred by dairy farmers in the present study. However with concerns over antimicrobial resistance, developing effective and safer alternatives to antimicrobial therapy in viral/bacterial infections is essential. Veterinarian was ranked the most credible source for treatment of dairy animals followed by paravet as evident from the preferential ranking by the dairy farmers. In this aspect, the veterinarians can do a useful job by treating the animals with those antimicrobials which has short withdrawal time. The paper also highlighted the nature of veterinary doctor antimicrobial prescribing decision and the choice of prescribed antimicrobial. Though the veterinarians relied more on prior experience for treating animals, sensitivity tests should also be conducted in choosing the right and most effective antimicrobial. Institutional facilities should be created at veterinary hospitals and dispensaries along with refresher training so as to facilitate sensitivity tests and aware the veterinarians regarding AMR issues in dairy sector. The existing uses of antimicrobial agents can be improved through increased utilization of veterinary professional services, the introduction of enhanced infection control measures, improved point-of-care diagnostic tests. Innovative research on dimensions of antimicrobial use in dairy farms and subsequent cost effective interventions coupled with primary stakeholders like farmers and veterinary doctors can pave way for tackling the AMR problem in a big way.

\section{Acknowledgements}

The first author acknowledges the contribution of Director, ICAR-NDRI, Karnal by providing institute fellowship for carrying out the research. The authors also thank the support and data provided by dairy farmers and veterinary doctors from study area of Punjab.

\section{Reference}

Aalipour F, Mirlohi M, Jalali M (2013) Prevalence of antibiotic residues in commercial milk and its variation by season and thermal processing methods. Int J Env Health Engg 2: 1-5

Bergevoet, RHM (2019) Economics of antibiotic usage on Dutch farms: The impact of antibiotic reduction on economic results of pig and broiler farms in the Netherlands (No. 019-026). Wageningen Econ Res.

Chand N, Sirohi AS, Tyagi S, Sharma A, Kumar S, Raja TV (2016) Comparative efficacy of homeopathic and allopathic treatments 
against Foot and Mouth disease in cattle. Indian J Anim Res 52: 898-902.

De Briyne N, Atkinson J, Pokludova L, Borriello SP, Price S (2013) Factors influencing antibiotic prescribing habits and use of sensitivity testing amongst veterinarians in Europe. Vet Rec 173: 475

Eltayb A, Barakat S, Marrone G, Shaddad S, Stålsby LC (2012) Antibiotic use and resistance in animal farming: a quantitative and qualitative study on knowledge and practices among farmers in Khartoum, Sudan. Zoonoses Pub Health 59: 330-338.

Garrett HE, Woodworth RS (1969) Statistics in Psychology and Education, Vakils, Feffer and Simons Pvt. Ltd. Bombay, pp. 329.

Gibbons JF, Boland F, Buckley JF, Butler F, Egan J, Fanning S, ... Leonard FC (2012) Influences on antimicrobial prescribing behaviour of veterinary practitioners in cattle practice in Ireland. Vet Rec 172: 14

Ison SH, Rutherford KMD (2014) Attitudes of farmers and veterinarians towards pain and the use of pain relief in pigs. The Vet J 202: 622627

Jones PJ, Marier EA, Trantera RB, Wub G, Watson E, Teale CJ (2015) Factors affecting dairy farmers attitudes towards antimicrobial medicine usage in cattle in England and Wales. Prev Vet Med 121:30 40

Kakkar M, Walia K, Vo ng S, Chatterjee P, Sharma, A (2017) Antibiotic resistance and its containment in India. Brit Med J 358: 2687

Kuralayanapalya SP, Patil SS, Hamsapriya S, Shinduja R, Roy P, Amachawadi RG (2019) Prevalence of extended-spectrum betalactamase producing bacteria from animal origin: A systematic review and meta-analysis report from India. PLoS ONE 14: e0221771

Kurjogi M, Issa Mohammad YH, Alghamdi S, Abdelrahman M, Satapute P, Jogaiah S (2019) Detection and determination of stability of the antibiotic residues in cow's milk. PLoS ONE 14: e0223475

Lindahl JF, Deka RP, Melin D, Berg A, Lunden H, Lapar ML, ... Grace, D (2018) An inclusive and participatory approach to changing policies and practices for improved milk safety in Assam, northeast India. Glob Food Sec 17: 9-13
Moudgil P, Bedi JS, Aulakh RS, Gill JPS (2019) Antibiotic residues and mycotoxins in raw milk in Punjab (India): a rising concern for food safety. J Food Sci Technol 56: 5146-5151

Nair BN, Natesan NP, Kempanna KS (2015) Role of ethno-veterinary practices (EVP) in reducing antimicrobial resistance in livestock production systems: A field experience. Plan Med 81: 3-6.

National Action Plan on Antimicrobial Resistance (NAP-AMR) 20172021 (2017). Government of India

National Health Policy (2017) Ministry of Health and Family Welfare. Government of India

Norris JM, Zhuo A, Govendir M, Rowbotham SJ, Labbate M, Degeling C (2019) Factors influencing the behaviour and perceptions of Australian veterinarians towards antibiotic use and antimicrobial resistance. PLoS ONE 14: e0223534

O’Neill J (2018) Tackling drug-resistant infections globally: Final report and recommendations. HM Government and Welcome Trust: UK

Patnaik NM, Gupta J, Acharya P, Kar P (2019) Use of Antimicrobials For Treatment of Dairy Animals by Veterinarian and Paravet in Punjab: A Study on Prescription Pattern. Indian J Ext Educ 55: 86-91

Sandholm M, Kaartinen L, Pyorala S (2009) Bovine mastitis why does antibiotics therapy not always work: An overview. J Vet Pharma Therap 13: $248-260$

Sawant AA, Sordillo LM, Jayarao BM (2005) A Survey on Antibiotic Usage in Dairy Herds in Pennsylvania. J Dairy Sci 88: 2991-2999

Sharma C, Rokana N, Chandra M, Singh BP, Gulhane RD, Gill JPS, Ray P, Puniya AK Panwar H (2018) Antimicrobial Resistance: Its Surveillance, Impact, and Alternative Management Strategies in Dairy Animals. Fron Vet Sci 4: 237 